\title{
Sex-related survival differences in murine cardiomyopathy are associated with differences in TNF-receptor expression
}

\author{
Toshiaki Kadokami, Charles F. McTiernan, Toru Kubota, \\ Carole S. Frye, and Arthur M. Feldman
}

Cardiovascular Institute of the University of Pittsburgh Medical Center Health System, University of Pittsburgh Medical Center, Pittsburgh, Pennsylvania, USA

Address correspondence to: Arthur M. Feldman, The Cardiovascular Institute of the University of Pittsburgh Medical Center Health System, 200 Lothrop Street, S 572 Scaife Hall, Pittsburgh, Pennsylvania 15213, USA. Phone: (412) 647-2769; Fax: (412) 647-3913; E-mail: feldmanam@msx.upmc.edu.

Received for publication January 5, 2000, and accepted in revised form July 14, 2000.

Epidemiological evidence suggests that the prognosis of heart failure in women is better than in men. In our murine model of dilated cardiomyopathy arising from cardiac-specific overexpression of TNF- $\alpha$, the 6-month survival rate was significantly better in females than in males. Young female transgenic mice exhibited left ventricular wall thickening without dilatation, whereas age-matched male transgenic hearts were markedly dilated. Basal and isoproterenol-stimulated fractional shortening was preserved in female transgenic mice, but not in male transgenic mice. Myocardial expression of proinflammatory cytokines and the extent of myocardial infiltrates were similar in male and female transgenic mice. Myocardial expression of TNF-receptor mRNAs (type I and type II) was significantly higher in male mice in both transgenic and wild-type littermates, whereas sex-specific differences were not observed in either peripheral white blood cells or liver tissue. After TNF- $\alpha$ challenge, myocardial but not liver production of ceramide was significantly higher in male than in female mice. Thus, differential expression of myocardial TNF receptors may contribute to sex differences in the severity of congestive heart failure and mortality consequent to cardiac-specific overexpression of TNF- $\alpha$.

J. Clin. Invest. 106:589-597 (2000).

\section{Introduction}

Epidemiologic studies have observed important differences in survival between men and women with heart failure (1-5). For example, the population-based Framingham Heart Study found that after the onset of symptomatic heart failure, the prognosis of women was significantly better than men $(3,4)$. Similar observations were reported in patients with advanced heart failure, and these sex-related differences could not be attributed to a high incidence of coronary disease in men $(1,2)$. Furthermore, hormone-replacement therapy appeared to effect improved survival in postmenopausal women with nonischemic heart failure (6).

Sex-related differences in the physiologic response to stress have also been identified in spontaneously hypertensive rats (7), transgenic mice overexpressing the cardiac $\mathrm{Na}^{+} / \mathrm{Ca}^{2+}$ exchanger (8), and rats with pressure overload-induced hypertrophy (9). However, studies in these animal models of heart failure have not assessed sexrelated survival nor have they elucidated underlying mechanisms that could account for these differences.

Recently, we developed transgenic mice (TNF1.6) with cardiac-specific overexpression of TNF- $\alpha$ and have characterized their progression to congestive heart failure $(10,11)$. These transgenic mice developed ventricular hypertrophy, cardiac dilatation, interstitial infiltrates and fibrosis, attenuation of adrenergic responsiveness, and re-expression of atrial natriuretic factor in the ventricle. These mice had a 6-month mortality of nearly $25 \%$. Furthermore, the mice that died spontaneously demonstrated exceptional dilatation of the heart, organized atrial thrombus, and massive pleural effusion, suggesting that they died of congestive heart failure (10). These characterizations have been confirmed by a separate group studying a similar transgenic model $(12,13)$. In this study we report the presence of sex-related differences in survival of TNF1.6 mice and have begun to elucidate cellular pathways that might be of etiologic importance in affecting sexrelated differences.

\section{Methods}

TNF- $\alpha$ transgenic mice. Studies used previously characterized transgenic mice (TNF1.6) (10). For RNA and protein analysis, animals were sacrificed, and excised ventricle or liver tissues were snap-frozen in liquid nitrogen. Blood samples were collected, and peripheral white blood cells were isolated by lysing erythrocytes with $1.7 \% \mathrm{NH}_{4} \mathrm{Cl}$. The mice were handled according to protocols approved by the Institutional Animal Care and Use Committee, University of Pittsburgh.

Experimental design. Survival analysis was used to assess differences between male and female transgenic mice. Echocardiography was performed to compare the cardiac structure and function between the sexes in vivo. To assess whether phenotypic differences arose from differ- 
ences in transgene expression or the differential expression of "downstream" cytokines or cytokine receptors, myocardial cytokine and cytokine-receptor expression was examined by RNase protection assay and ELISA. To determine whether differential myocardial expression of TNF receptor results in differential second-messenger production, myocardial ceramide production in response to acute treatment with TNF- $\alpha$ was assayed in both male and female FVB mice.

Echocardiographic measurements. Echocardiographic studies were performed with an Acuson ultrasonograph system (Sequoia 512; Acuson, Mountainview, California, USA). Mice were anesthetized with intraperitoneal injection of $2.5 \%$ Avertin $(18 \mu \mathrm{L} / \mathrm{g}$ body weight) (14) and were lightly secured in a shallow, left-lateral decubitus position. A 13.0-MHz transducer was applied to the left hemithorax. Two-dimensional targeted M-mode imaging was obtained from the short-axis view at the level of the largest left ventricular diameter at baseline and after the intraperitoneal administration of isoproterenol (300 ng/g body weight). M-mode measurements of left ventricular end-diastolic and end-systolic diameter and left ventricular anterior- and posterior-wall thickness were made from images recorded on videotape using the leading-edge convention of the American Society of Echocardiography. Three to five beats were averaged for each measurement. End diastole was determined at the maximal left ventricular diastolic dimension, and end systole was taken at the peak of posterior-wall motion. Heart rate was determined from three to five consecutive $\mathrm{R}$ wave intervals determined from electrocardiogram. The percentage of left ventricular fractional shortening (LVFS) was calculated as: LVFS (\%) = [(LVEDD - LVESD)/LVEDD] $\times 100$, where LVEDD and LVESD indicate left ventricular end-diastolic and endsystolic diameter, respectively.

RNase protection assay. Total RNA was extracted from frozen tissues as described $(10,11)$. Transcript levels of cytokines and cytokine receptors in myocardium, peripheral white blood cells, and liver were examined by multiprobe RNase protection assay (RPA) performed according to the manufacturer's protocol (RiboQuant [PharMingen, San Diego, California, USA], using templates mCR-4 [No. 45364P], and a custom mouse template set [No. 557310] containing probes for TNF- $\alpha$, IL6 , IL-1 $\beta$, TGF- $\beta 1$, MCP- 1 , L32, and GAPDH). After interaction of radiolabeled probes with $5 \mu \mathrm{g}$ of total RNA and RNase digestion, the protected probes were purified, resolved on denaturing polyacrylamide gels, and quantified by PhosphoImager using ImageQuant software (Molecular Dynamics, Sunnyvale, California, USA). The value of each hybridized probe was normalized to that of GAPDH included in each template set as an internal control (arbitrarily set at 1 ).

ELISA. Cytokine protein levels were assessed using ELISA kits for mouse TNF- $\alpha$, mouse IL- $1 \beta$, and mouse MCP-1 (Quantikine; R\&D Systems, Minneapolis, Minnesota, USA). Frozen tissues (5-20 mg) were homogenized in 300-500 $\mu \mathrm{L}$ ice-cold PBS containing $1 \mathrm{mmol} / \mathrm{L}$ phenylmethylsulfonyl fluoride protease inhibitor (Sigma Chemical Co., St. Louis, Missouri, USA). After brief centrifugation, samples were kept on ice for the duration of the assay. Total protein levels were quantified (Bio-Rad Protein Assay; Bio-Rad Laboratories Inc., Hercules, California, USA) with BSA (Sigma Chemical Co.) as a standard. Cytokines in the myocardium were measured using $100 \mu \mathrm{g}$ of protein for TNF- $\alpha$, IL- $1 \beta$, and MCP-1 (15). Standard reference cytokines were provided by the manufacturer. Assays were done in duplicate with a microtiter plate reader (450-nm wavelength). Values are reported as picograms per milligram of protein.

Quantitation of myocardial infiltration. Hearts were fixed in ice-cold $2 \%$ paraformaldehyde, immersed in ice-cold $30 \%$ sucrose for 2 hours, and then flash-frozen in OCT media using isopentane cooled by liquid nitrogen. Blocks were cut on a cryostat at $10 \mu \mathrm{m}$ and sections were mounted and subjected to hematoxylin and eosin staining. To quantitate myocardial infiltration, nuclear density (nuclei $/ \mathrm{mm}^{2}$ ) was determined. Since it is difficult to differentiate inflammatory cells from myocytes and/or fibroblasts, all nuclei were included to obtain nuclear density. In each animal, six independent highpower fields $\left(0.233 \mathrm{~mm} \times 0.312 \mathrm{~mm} ; 0.0729 \mathrm{~mm}^{2}\right.$ area $)$ were analyzed and averaged by investigators blinded to the treatment group.

Ceramide assay. Cardiac and hepatic ceramide production in response to TNF- $\alpha$ stimulation was measured according to the modified method of Preiss et al. (16). Briefly, FVB mouse left-ventricular and liver tissue was isolated from both sexes and immediately cut into $\sim 2 \times$ $1 \times 1$-mm strips. The tissue strips were equilibrated in M199 medium without supplements and stimulated for 30 minutes with either TNF- $\alpha(200 \mathrm{U} / \mathrm{mL}$ recombinant rat; BioSource International, Camarillo, California, USA) or PBS, with or without $n$-oleoylethanolamine (NOE; 1 $\mathrm{mM}$; Sigma Chemical Co.) (17). Tissue samples were homogenized in $1 \mathrm{M} \mathrm{NaCl}$, and lipid fractions were extracted with chloroform/methanol (1:2 vol/vol). The aqueous phase was used for determination of the protein concentration, whereas the chloroform phase was dried in vacuo. The extracts were resuspended and samples corresponding to $1 \mu \mathrm{g}$ protein were used in a diacylglycerol (DAG) kinase assay. To assess the relationship between recovered phospholipid and tissue-sample protein content, additional cardiac tissue samples were used to measure total phospholipid content by the method of Hess and Derr (18), and the correlation between total phospholipid and protein content of the samples was determined. Samples were incubated with Escherichia coli DAG kinase and $\left[\gamma^{32} \mathrm{P}\right]$ ATP using a commercially available kinase assay system (Biotrack No. RPN200; Amersham Pharmacia Biotech, Piscataway, New Jersey, USA). Experimental samples and a parallel ceramide 1-phosphate standard ( $1 \mu \mathrm{g}$; Sigma Chemical Co.) were spotted on TLC plates (LHPKDF silica gel, $60 \AA$; Whatman International, Maidstone, United Kingdom) and developed in chloroform/methanol/acetic acid (65:15:5 $\mathrm{vol} / \mathrm{vol} / \mathrm{vol})$. Nonradioactive ceramide 1-phosphate, visu- 
alized by spraying plates with $10 \%$ $\mathrm{CuSO}_{4} / 8 \% \mathrm{H}_{3} \mathrm{PO}_{4}$ followed by charring $\left(180^{\circ} \mathrm{C}\right.$ for 10 minutes $)$, demonstrated a fractional mobility relative to that of the solvent front (Rf) of 0.35 , identical to that of the radioactive ceramide 1-phosphate standard generated by application of the DAG kinase reaction to nonradiolabeled ceramide. Radioactive images were identified by PhosphoImager and quantified using ImageQuant software (Molecular Dynamics). In an additional experiment, dose responses of cardiac ceramide production were determined in the presence of NOE $(1 \mathrm{mM})$ in tissue samples obtained from six female and six male mice. In this case, three different doses of TNF- $\alpha$ were used $(20,200$, and 1,000 $\mathrm{U} / \mathrm{mL}$ ). Tissue strips incubated with neither TNF- $\alpha$ nor NOE served as controls, with their value arbitrarily set at $100 \%$. Tissue production of ceramide was expressed as the percentage of control values.

Statistics. Results are presented as mean plus or minus SD (unless specified otherwise). Statistical comparisons were performed using ANOVA with Student-NewmanKeuls post-hoc test. Differences were considered significant at a value of $P<0.05$. Kaplan-Meier survival analysis was used to assess all causes of mortality in the different groups separated by genotype or sex.

\section{Results}

Sex-specific survival difference in TNF1.6 mice. Survival analysis shows marked difference between male $(n=$ $158)$ and female $(n=401)$ transgenic mice $(P<0.001)$ (Figure 1) up to 1 year of age. By 6 weeks of age, the survival curve of male TNF1.6 mice starts to decline and continues to decrease until more than $80 \%$ of the mice died from heart failure at 70 weeks of age. Only $4 \%$ of TNF1.6 females died by the age of 21 weeks, at which point the survival curve decreased gradually. The 6month survival rate of male and female TNF1.6 mice was $52 \%$ and $89 \%(P<0.001)$, respectively. There was no significant survival difference in male and female wildtype mice; however, the life expectancy of a wild-type mouse substantially exceeds 1 year (Figure 1 ). In subsequent experiments we used either 6-week-old (echocardiography), 8-week-old (RNase protection assay and ELISA), or 12-week-old (ceramide mass assay) mice. These time points were selected from the age interval at which the survival curves of male and female TNF1.6 mice started to separate.

Sex-specific difference in left ventricular structure and function in TNF1.6 mice. Results of echocardiography of TNF1.6 mice and wild-type littermates of both sexes (6 weeks of age) are shown in Figure 2 and Table 1. Male TNF1.6 mice exhibited marked left ventricular dilatation and relatively thin walls, with reduced fractional shortening. Left ventricular fractional shortening failed to significantly increase after isoproterenol challenge (Fig- ure 2i). By contrast, left ventricular wall thickness but not chamber dimension was increased in female TNF1.6 mice, with diminished but persistent isoproterenol responsiveness. Although the left ventricular dimension of wild-type mice was slightly but significantly larger in males than females, these values were equivalent after normalization for body weight (Table 1). In wild-type mice, both sexes displayed a comparable increase in fractional shortening in response to isoproterenol challenge. Despite the substantial decrease in fractional shortening, there were no significant changes in heart rate under anesthesia between TNF1.6 and wild-type mice. This is consistent with our previous observations (10). Furthermore, ambulatory conscious telemetry monitoring of electrocardiographs on TNF1.6 mice (B. London, University of Pittsburgh, unpublished data) showed no significant differences in heart rate between TNF1.6 and wild-type mice.

Ventricular weight and ventricular weight/body weight ratio of TNF1.6 mice and wild-type controls are summarized in Table 2. Ventricular weight normalized by body weight was significantly larger in TNF1.6 mice relative to wild-type mice, confirming our previous observations $(10,15)$, as well as in male TNF1.6 mice compared with female TNF1.6 mice. We also determined that there was an excellent correlation between

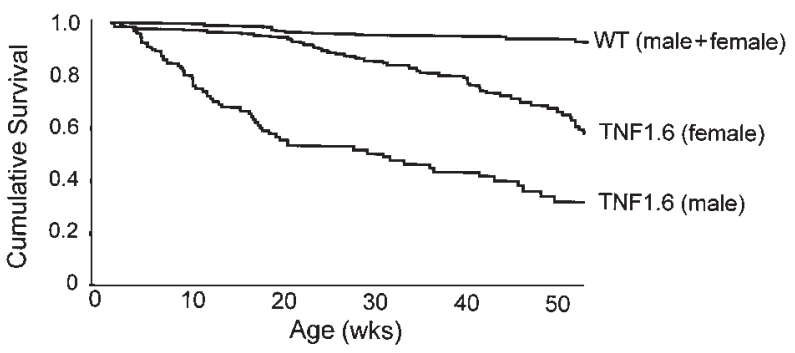

Figure 1

Survival-function curve ofTNF1.6 mice $(n=559)$ and wild-type littermates $(n=842)$. Female TNF1.6 mice $(n=401)$ survive significantly longer than male TNF1. 6 mice $(n=158 ; P<0.001)$. Mean survival time (limited to 360 days) for wild-type mice, female TNF1. 6 mice, and male TNF1. 6 mice was $346 \pm 4,286 \pm 8$, and $179 \pm 12$ days, respectively (mean $\pm \mathrm{SE}$ ). 
Figure 2

Echocardiographic studies in TNF1.6 mice. (a-h) Representative images of M-mode echocardiogram of TNF1.6 mice (a-d) and wild-type littermates (e-h). Male TNF1.6 mice exhibited a markedly dilated left ventricle with reduced fractional shortening (a) and poor responsiveness to isoproterenol (b). Female TNF1.6 mice showed hypertrophied but not dilated left ventricle (c) with relatively preserved isoproterenol responsiveness (d). Both male and female wild-type mice had good left ventricular systolic function and showed a significant increase in fractional shortening after isoproterenol challenge $(\mathbf{e}-\mathbf{h})$. (i) Left ventricular fractional shortening before (filled bars) and after (open bars) isoproterenol injection in TNF1. 6 and wild-type mice. Wild-type mice of both sexes showed preserved systolic function at baseline measurement, and fractional shortening was significantly increased up to $40 \%$ after isoproterenol challenge. By contrast, male TNF1. 6 mice exhibited decreased fractional shortening at baseline and completely lost the responsiveness to $\beta$-adrenergic stimulation. Basal left ventricular contraction was preserved in female TNF1.6 mice, whereas the increase in fractional shortening after isoproterenol injection was significant but impaired. Values are mean \pm SD. TG, TNF1.6 mice; WT, wild-type mice; M, male; F, female; FS, fractional shortening; NS, not significant. ${ }^{A} P<0.05$ vs. WT; ${ }^{B} P<0.01$; ${ }^{C} P=0.001$. (j) Correlation of left ventricular mass estimated from echocardiographic measurements and gravimetric mass determination. A total of 16 TNF1.6 or wild-type mice were sacrificed immediately after echocardiographic examination, hearts were excised, and left ventricles were trimmed and weighed. Left ventricular mass was calculated using the formula in the text. Left ventricular mass determined by the two different methods showed an excellent correlation $(P<0.001)$.

the left ventricular mass calculated from echocardiography and gravimetric mass determination (Figure 2j; $P$ $<0.001, n=16)$ obtained at the time of sacrifice. Thus, echocardiography provided an accurate measure of cardiac mass. The left ventricular mass calculated from echocardiography was also larger in TNF1.6 mice relative to wild-type littermates and in male TNF1.6 mice relative to female TNF1.6 mice (Table $1 ; P<0.001$ ).

Expression of cytokines and extent of myocardial infiltrates. Since in this model, cardiac dysfunction and subsequent heart failure are induced through chronic overexpression of TNF- $\alpha$, it was important to assess potential sex-related differences in the expression of TNF- $\alpha$. Analysis by both RPA and ELISA demonstrated markedly elevated expression of TNF- $\alpha$ without significant differences between male and female mice (Figure 3). In addition, overexpression of TNF- $\alpha$ enhances expression of a variety of other cytokines (15) that may contribute to cardiac pathology. For example, the proinflammatory cytokine IL- $1 \beta$ typically works synergistically with TNF- $\alpha$, but has also been shown to have important effects on cardiomyocyte function independent of TNF- $\alpha$ (19-21). MCP-1, which may mediate myocardial infiltration, can produce heart failure when over-expressed in the myocardium (22). TGF- $\beta$ may contribute to cardiac fibrosis (23) as well as hypertrophy (24). To assess whether sexspecific differences in the expression of these downstream cytokines underlie the differences in survival and cardiac pathology, we examined myocardial expression of TNF- $\alpha$, IL- $1 \beta$, TGF- $\beta 1$, and MCP- 1 transcripts by multiprobe RPA, and used ELISA assays to examine cardiac expression of TNF- $\alpha$, IL- $1 \beta$, and MCP- 1 proteins. Similar to TNF- $\alpha$, IL-1 $\beta$ and MCP- 1 transcripts and proteins were markedly and similarly elevated in TNF1.6 mice of both sexes. Gene expression of TGF- $\beta 1$ was also upregulated in TNF1.6 mice (Figure 3; $n=6$, 8-week-old mice), consistent with our previous observations (15).

Table 2

Body and ventricular weights of TNF- $\alpha$ transgenic mice

\begin{tabular}{lcccc}
\hline & \multicolumn{2}{c}{ TNF1.6 } & \multicolumn{2}{c}{ Wild-type } \\
& Male & Female & Male & Female \\
$n$ & 9 & 10 & 9 & 9 \\
BW, g & $20.3 \pm 4.3$ & $19.4 \pm 1.1$ & $23.2 \pm 1.4^{\mathrm{B}}$ & $20.7 \pm 1.9$ \\
WW, mg & $120.0 \pm 28.7^{\mathrm{A}, \mathrm{B}}$ & $94.8 \pm 8.0^{\mathrm{A}}$ & $94.7 \pm 10.0^{\mathrm{B}}$ & $82.7 \pm 4.0$ \\
WW/BW, mg/g & $5.90 \pm 0.49^{\mathrm{A}, \mathrm{B}}$ & $4.91 \pm 0.50^{\mathrm{A}}$ & $4.07 \pm 0.28$ & $4.03 \pm 0.24$
\end{tabular}

$\mathrm{BW}$, body weight; $\mathrm{WW}$, ventricular weight. Values are mean $\pm \mathrm{SD}$. ${ }^{A} P<0.05 \mathrm{vs}$. wild-type of the same sex. ${ }^{B} P<0.05$ vs. female of the same genotype. 
Another consequence of cardiac-specific overexpression of TNF- $\alpha$ is an increase in the number of infiltrating cells in the myocardium. These infiltrating cells may express cytokines or other factors that influence cardiac structure and function, thus differences in the severity of cardiac pathology could arise from differences in the extent of myocardial infiltrates. To assess the level of myocardial infiltrates, the nuclear density of myocardium from 8-week-old animals was determined and shown to be comparable in male and female TNF1. 6 mice $\left(7572 \pm 1289\right.$ nuclei $/ \mathrm{mm}^{2}, 7010 \pm 796$ nuclei $/ \mathrm{mm}^{2}$, respectively; $n=5$ ), although TNF1.6 mice exhibited markedly higher nuclear density than wildtype males and females (3306 \pm 302 nuclei/ $/ \mathrm{mm}^{2}, 3155$ \pm 219 nuclei $/ \mathrm{mm}^{2}$, respectively; $n=5, P<0.05$ ).

Expression of cytokine receptors in myocardium and other organs. Sex-specific differential responses to similar levels of cytokine expression could arise from differences in the expression of cytokine receptors. Myocardial gene expression of various cytokine receptors, including TNFR1 and TNFR2, was examined by multiprobe RPA (8-week-old mice, $n=6$ in each group). Transcript levels of TNFR1 were significantly higher in TNF1. 6 relative to wild-type mice, and male mice exhibited a twofold higher level relative to female mice in both TNF1.6 and wild-type animals (Figure 4). Similarly, the transcript level of TNFR2 was fivefold higher in male animals rel- ative to female animals, whether transgenic or wild-type. By contrast, there was no sex difference in transcript levels for the TGF- $\beta$-receptor groups and gp 130 .

Cytokine-receptor expression was also examined in peripheral white blood cells (8-week-old mice, $n=6$ in each group) and liver tissues (8-week-old mice, $n=5$ in each group). In peripheral white blood cells, TNF1.6 mice of both sexes expressed significantly more TNF receptors when compared with wild-type animals of the same sex, but the extent of expression was comparable between sexes (Figure 5). On the other hand, in liver tissue there was no significant difference in the expression of any of the cytokine-receptor transcripts between either sex or genotype.

Ceramide production in response to TNF stimulation. Measurements of steady-state levels of mRNA can be misleading because mRNA levels may not correlate with protein quantity. However, since mouse-specific Ab's having adequate sensitivity for quantitative analysis of TNF-receptor expression were not readily available, assessment of the relevance of the decrease in steadystate levels of TNFR mRNA proved challenging. To demonstrate that the difference in TNFR mRNA was of physiologic significance, we measured the ability of TNF- $\alpha$ to elicit the production of the TNF- $\alpha$-responsive second messenger molecule, ceramide. Myocardialtissue strips were transiently cultured, exposed to TNF- a

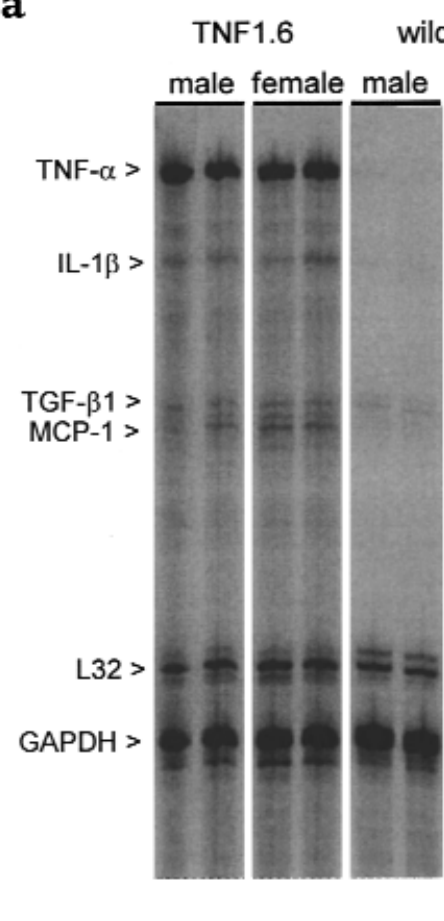

b

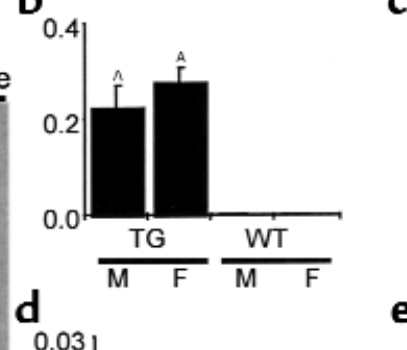

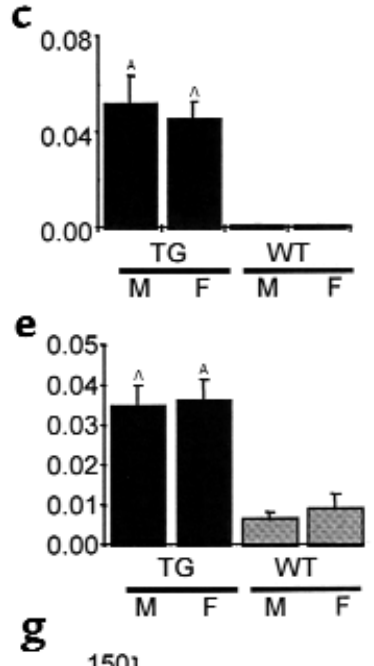

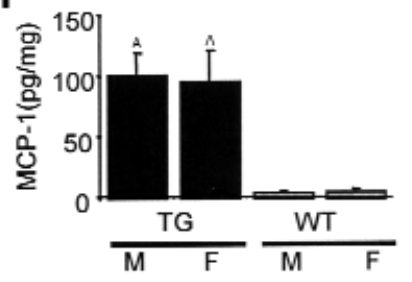

Figure 3

Cytokine expression in the myocardium. (a-e) Representative images (a) and quantitative results of RNase protection assays for TNF- $\alpha(\mathbf{b}), \mathrm{IL}-1 \beta(\mathbf{c})$, $\mathrm{MCP}-1$ (d), and TGF- $\beta 1$ (e). Quantitative results are reported as the ratio

of the pixel intensity of each protected probe normalized to that of the GAPDH probe included in each template set as an internal control, as described in Methods. Although TNF1.6 mice expressed significant amounts of TNF- $\alpha$ and other cytokines, the transcript levels of TNF- $\alpha$ and other downstream cytokines were not significantly different between male and female mice. $Y$-axis values correspond to the signal intensity of the hybridized probe in pixels normalized to that of GAPDH. (f-h) Results of ELISA for TNF- $\alpha(\mathbf{f})$, IL-1 $\beta(\mathbf{g})$, and MCP-1 (h) showed significantly elevated levels in TNF1.6 versus wild-type mice, but no difference between sexes of the same genotype. Values are mean $\pm \mathrm{SD}(n=6)$. TG, TNF1.6 mice; WT, wild-type mice; M, male; F, female. ${ }^{A} P<0.001$ vs. WT of the same sex.

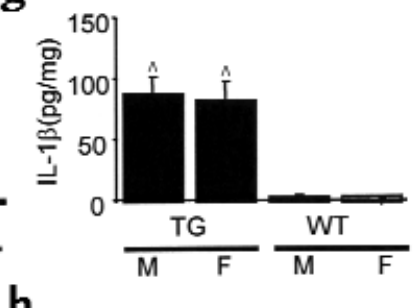

h

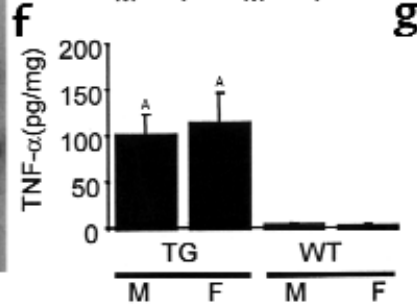

1
6


Figure 4

Cytokine-receptor expression in myocardium. Representative images (a) and quantitative results of RNase protection assays for TNFR1 (b) and TNFR2 (c). Quantitative results are reported as the ratio of the pixel intensity of each protected probe normalized to that of the GAPDH probe included in each template set as an internal control, as described in Methods. TNF1.6 mice expressed significantly more TNF receptors. In addition, the transcript level of TNF receptors was significantly higher in males relative to females in both TNF1.6 and wild-type mice. Values are mean \pm SD $(n=6) . Y$-axis values correspond to the signal intensity of the hybridized probe in pixels normalized to that of GAPDH. TG, TNF1.6 mice; WT, wild-type mice; $M$, male; $F$, female. ${ }^{A} P<0.001$ vs. females of the same genotype. ${ }^{B} P<0.01 \mathrm{vs}$. wild-type of the same sex. a

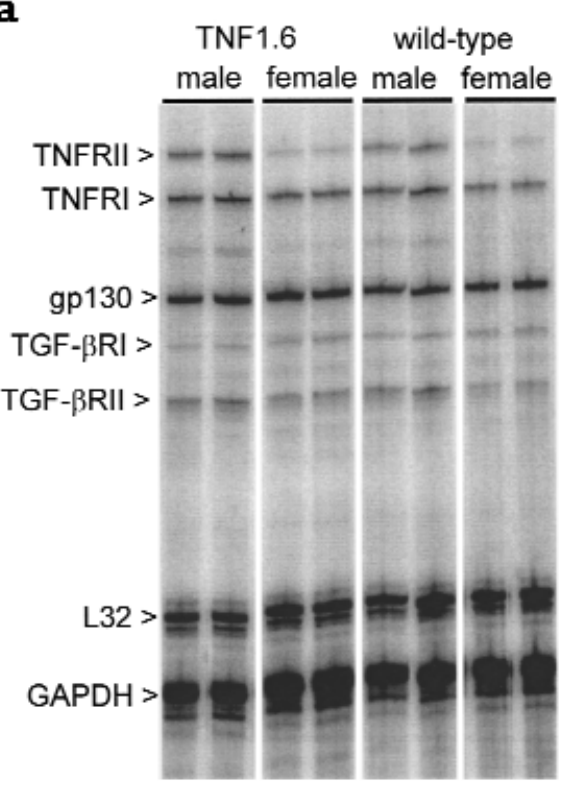

b

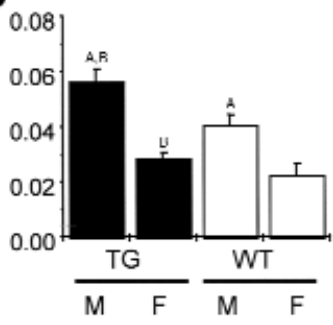

c

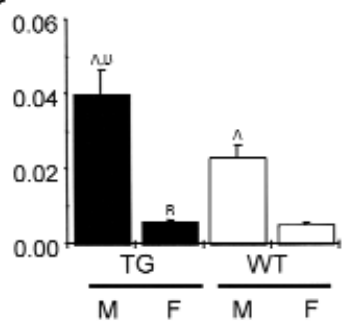

$\alpha$, and the production of ceramide was determined by a DAG kinase assay. Exposure of tissue strips to TNF$\alpha$ induced considerable production of ceramide, which was detectable (up to twofold increase compared with PBS-treated control) in both sexes. In fact, tissue strips incubated without TNF- $\alpha$ stimulation produced a significant amount of ceramide in a time-dependent manner (data not shown), suggesting that the culture system itself is a stimulus of ceramide production. However, the ceramide-production capacity should be largely preserved because we observed further increase in ceramide production upon TNF- $\alpha$ stimulation. Fur- thermore, we could detect much more ceramide in the tissue strips treated with TNF- $\alpha$ in the presence of NOE, a ceramidase inhibitor, and the amount of detected ceramide was significantly higher in male, relative to female, mice (male/female $=720 \pm 330 \%: 410 \pm$ $225 \%, n=5 ; P<0.05)$, suggesting that the response to TNF- $\alpha$ was significantly enhanced in male hearts relative to female hearts (Figure 6, a and b). Interestingly, in cardiac tissues the ceramide content appears larger than that of DAG, consistent with a previous report that used a different assay method (25). To confirm the finding that levels of expression of the mRNA encod- a

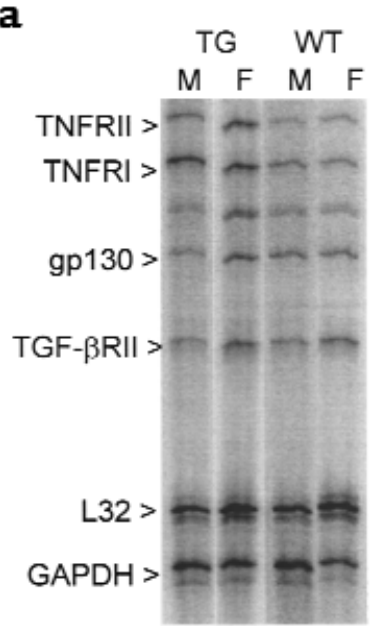

b

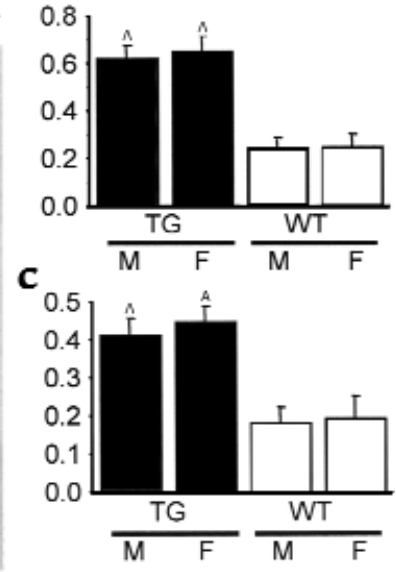

d

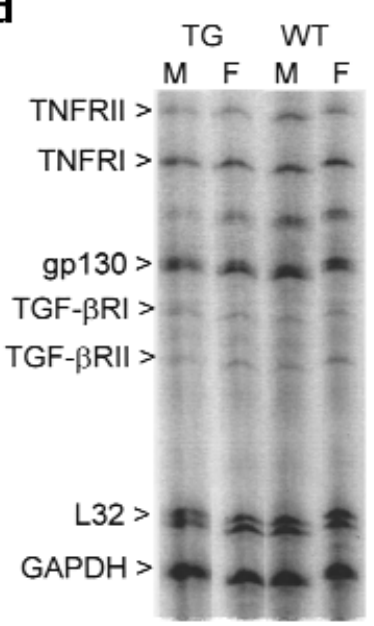

e

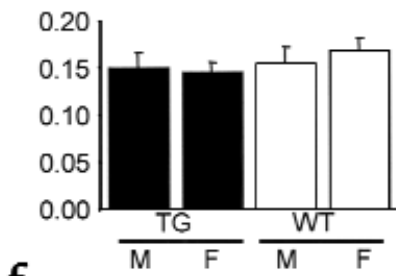

f

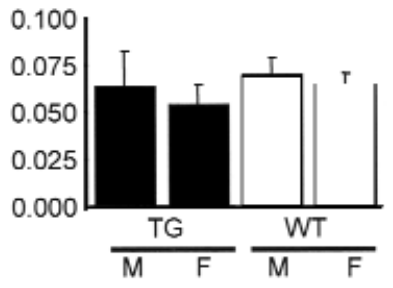

Figure 5

Cytokine-receptor expression in peripheral white blood cells and liver tissues. Representative images and quantitative results of RNase protection assays for TNFR1 and TNFR2 in peripheral white blood cells $(\mathbf{a}-\mathbf{c})$ and liver tissues $(\mathbf{d}-\mathbf{f})$. Quantitative results are reported as the ratio of the pixel intensity of each protected probe normalized to that of the GAPDH probe included in each template set as an internal control, as described in Methods. Although TNF1.6 mice expressed significantly more TNFR1 and TNFR2 RNA in peripheral white blood cells, there was no significant difference between sexes in cytokine-receptor gene expression in TNF1.6 and wild-type mice (a-c). Transcript level of cytokine receptors in the liver was comparable in males and females in both TNF1.6 and wild-type mice (d-f). Values are mean \pm SD. $Y$ axis values correspond to the signal intensity of the hybridized probe in pixels normalized to that of GAPDH. TG, TNF1.6 mice; WT, wildtype mice; $M$, male; F, female. ${ }^{A} P<0.001$ vs. WT of the same sex. 
ing TNF receptors did not differ in the liver, we also assessed the TNF- $\alpha$-induced synthesis of ceramide in male and female liver tissues (Figure 6, c and d). Ceramide production was clearly enhanced after exposure of liver tissues to TNF- $\alpha$ and was further elevated when studies were performed in the presence of NOE. However, unlike myocardial tissues, liver tissue from male and female mice produced equivalent levels of ceramide in response to TNF- $\alpha$ exposure $(n=5)$.

To further confirm the sex-specific differences in myocardial TNF- $\alpha$-induced ceramide production, we examined the ceramide production in response to three different doses of TNF- $\alpha$ in the presence of NOE $(1$ $\mathrm{mM})$. As shown in Figure 6e, male hearts produced significantly more ceramide than female hearts at both $200 \mathrm{U} / \mathrm{mL}$ and $1,000 \mathrm{U} / \mathrm{mL}$ of TNF- $\alpha(P<0.01, n=6)$. a

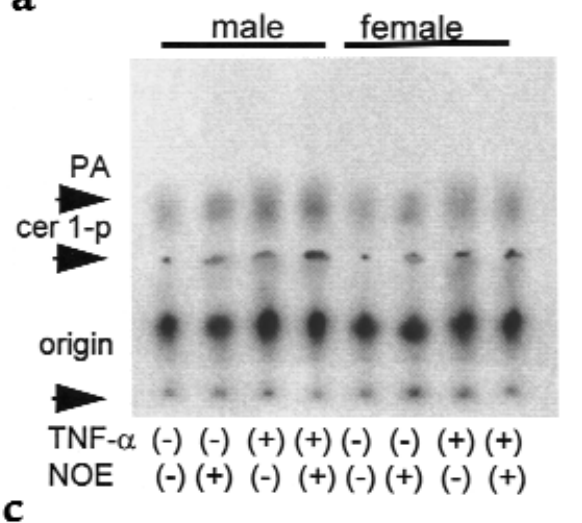

C

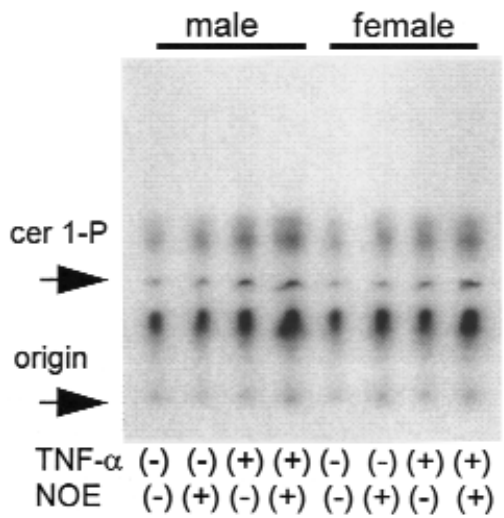

b

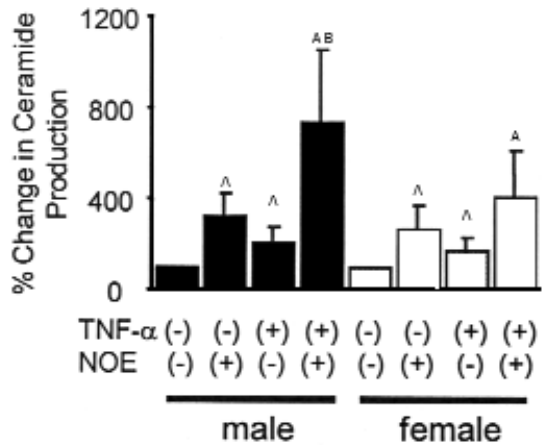

d

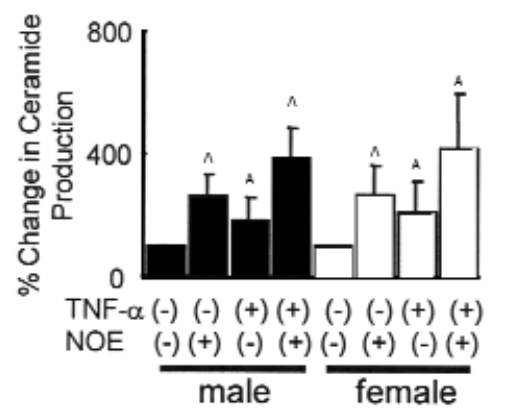

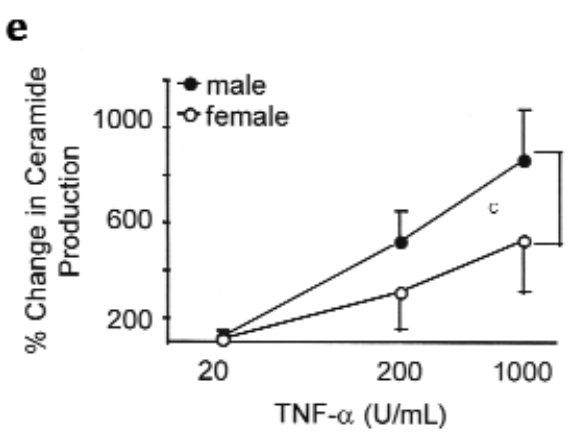
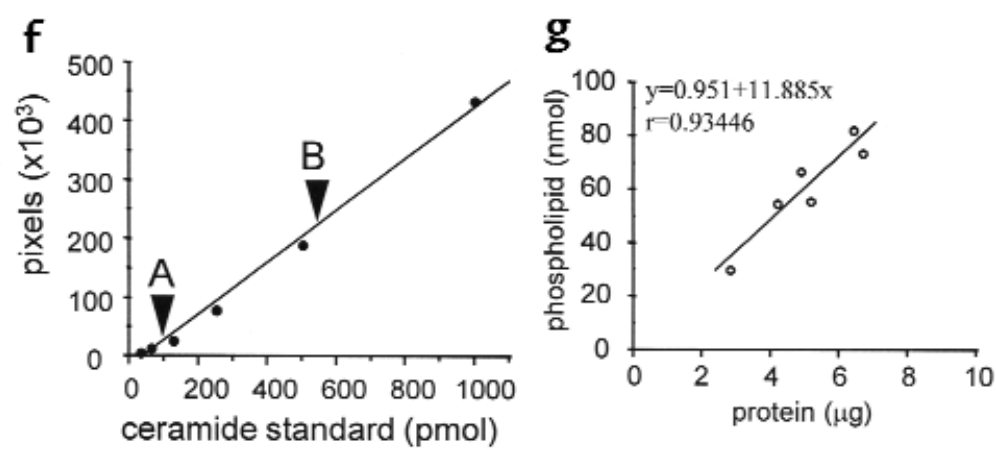

Figure 6

Ceramide production in response to acute TNF- $\alpha$ stimulation in FVB mice. Representative images (a) and quantitative results (b) of cardiac-tissue ceramide production. Detectable ceramide mass measured by DAG-kinase assay was significantly greater in male cardiac tissue than in female in the presence of both TNF- $\alpha$ and NOE. Although treatment with TNF- $\alpha$ or NOE alone resulted in significant increase in ceramide production, ceramide mass content was not significantly different between sexes in those groups. Representative images (c) and quantitative results (d) of liver-tissue ceramide production. By contrast to cardiac tissues, additional treatment with NOE plus TNF- $\alpha$ did not result in a significant difference in terms of ceramide production, although the treatment stimulated a substantial amount of ceramide production in both sexes. (e) Dose response of cardiac-tissue ceramide production in the presence of both TNF- $\alpha$ and NOE. Male hearts produced significantly more ceramide in response to both low $(200 \mathrm{U} / \mathrm{mL})$ and high $(1,000 \mathrm{U} / \mathrm{mL})$ doses of TNF- $\alpha$. (f) Standard curve of DAG-kinase assay. Note that both samples with low- (A: -TNF- $\alpha,-N O E)$ and high- (B: +TNF- $\alpha,+N O E)$ ceramide content fall within the linear range of the assay. (g) Correlation between total-protein and phospholipid content of the samples. A strong positive correlation was observed between tissue-protein content and phospholipid content $(P<0.005)$. Ceramide production was expressed as the percentage of control $(-\mathrm{TNF}-\alpha,-\mathrm{NOE})$ values, set at $100 \%$. Values are mean $\pm \mathrm{SD} .{ }^{A} P<0.01$ vs. control. ${ }^{\mathrm{B}} P<0.05$ vs. female $(+\mathrm{TNF}-\alpha,+\mathrm{NOE}) .{ }^{C} P<0.01$. 


\section{Discussion}

The cardiac-specific overexpression of TNF- $\alpha$ in transgenic mice effects the development of cardiac dilatation, myocyte hypertrophy, extracellular matrix remodeling, cytokine and chemokine re-expression, insensitivity to adrenergic stimulation, and a shortened survival $(10,15,26)$. In the present study, we demonstrate marked sex-specific differences in left ventricular function, findings that are consistent with earlier reports demonstrating sex-specific differences in hemodynamic parameters and gene expression in rodent models of load-induced heart failure (9). More importantly, we also report a substantial survival benefit in female mice having this well-characterized genetically-induced cardiomyopathy when compared with age-and litter-matched males. Recent studies in patients demonstrate that women with heart failure live substantially longer than men (1-3). However, we provide, to our knowledge, the first evidence of sexrelated survival benefits in an animal model of heart failure. Thus, transgenic mice overexpressing TNF- $\alpha$ may provide a relevant model in which to understand sex-related survival in humans.

The marked differences in survival in the male and female mice overexpressing TNF- $\alpha$ could not be attributed to differences in the levels of expression of TNF- $\alpha$ or to that of downstream cytokines and chemokines because the levels of TNF- $\alpha$ expression, both at the transcript and protein level, were identical in male and female mice. By contrast, male TNF1.6 mice had substantially higher steady-state levels of the mRNAs encoding both TNF receptors (TNFR1 and TNFR2). This sex-specific difference in the amount of receptor mRNA could also be identified in wild-type mice. Because steady-state mRNA levels may not correlate with those of the mature protein, we assessed the physiologic significance of this difference by taking advantage of the fact that ceramide production is TNF- $\alpha$ dependent. TNF- $\alpha$-induced oligomerization of TNFR1 activates membrane-bound neutral sphingomyelinase with subsequent production of ceramide (27). Thus, the finding that myocardial tissue from male mice produced significantly more ceramide than that of females provided pharmacologic and physiologic confirmation of the relevance of the enhanced expression of TNF receptors in male mice. Furthermore, there was a stoichiometric relationship between the difference in TNFR mRNA levels and TNF- $\alpha$-induced ceramide production in males and females. Taken together, these results suggest that enhanced survival in female mice in the presence of TNF- $\alpha$ overexpression may be attributable to sex-related differences in the expression of TNF receptors.

The results of the present study might lead one to suspect that differences in TNF receptor level and responsiveness might contribute to sex-related differences in a variety of different cardiovascular or noncardiac diseases. However, it is important to note that whereas the failing heart produces robust quantities of
TNF- $\alpha$, the normal heart does not produce TNF- $\alpha$. Thus, under baseline conditions the relevance of the sex-related differences in TNF-receptor expression remains unclear. Furthermore, we were unable to demonstrate sex-related differences in TNF- $\alpha$ responsiveness in hepatocytes or in white blood cells, precluding the possibility that overexpression of TNF- $\alpha$ by disease states in these organs could effect sex-related differences in hepatic or hematologic diseases. In addition, the finding that the amount of inflammatory infiltrates did not differ in male and female TNF1.6 mice obviates the possibility that differences in the number of leukocytes could have artifactually resulted in an increase of TNF-receptor mRNA in the myocardium of male mice.

Although the present results support the hypothesis that sex-related differences in survival in mice with heart failure secondary to TNF- $\alpha$ overexpression is attributable to enhanced expression of TNF receptors in males, the etiology of these differences remains unclear. Recent studies suggest that antiestrogen treatment enhanced TNFR2 expression and TNFR2-mediated proliferation in activated T cells (28). Furthermore, estrogen appears to have a protective role in the development of osteoporosis by limiting expression of IL-1 receptors (29). Finally, ovariectomy decreased the expression of TNFR1 in the mouse uterus (30). Therefore, estrogen may regulate cytokine-receptor expression in an organ-specific manner and be responsible for the survival advantage enjoyed by females, a hypothesis that would be consistent with the finding of improved survival in postmenopausal women receiving hormone-replacement therapy (6).

In summary, transgenic mice with heart failure secondary to overexpression of TNF- $\alpha$ demonstrate sexrelated phenotype differences in survival, ventricular function, and cardiac gene expression. These differences appear to be attributable to sex-related expression of TNF receptors within the myocardium. Additional studies are required to determine the cellular and molecular mechanisms responsible for the differential regulation of the TNF receptors.

\section{Acknowledgments}

T. Kadokami was supported by funds from the Bill Hillgrove Cardiology Fellowship. T. Kubota is the recipient of a Japan Heart Foundation \& Bayer Yakuhin Research Grant Abroad.

1. Adams, K.F., Jr., et al. 1996. Relation between gender, etiology and survival in patients with symptomatic heart failure. J. Am. Coll. Cardiol. 28:1781-1788.

2. Adams, K.F., Jr., et al. 1999. Gender differences in survival in advanced heart failure. Insights from the FIRST study. Circulation. 99:1816-1821.

3. Ho, K.K., Anderson, K.M., Kannel, W.B., Grossman, W., and Levy, D. 1993. Survival after the onset of congestive heart failure in Framingham Heart Study subjects. Circulation. 88:107-115.

4. McKee, P.A., Castelli, W.P., McNamara, P.M., and Kannel, W.B. 1971. The natural history of congestive heart failure: the Framingham study. N. Engl. J. Med. 285:1441-1446.

5. Carroll, J.D., et al. 1992. Sex-associated differences in left ventricular function in aortic stenosis of the elderly. Circulation. 86:1099-1107.

6. Reis, S.E., et al. 1998. Postmenopausal estrogen use predicts improved 
survival in women with congestive heart failure. Circulation 98:I205. (Abstr.)

7. Pfeffer, J.M., Pfeffer, M.A., Fletcher, P., Fishbein, M.C., and Braunwald E. 1982. Favorable effects of therapy on cardiac performance in spontaneously hypertensive rats. Am. J. Physiol. 242:H776-H784.

8. Cross, H.R., Lu, L., Steenbergen, C., Philipson, K.D., and Murphy, E. 1998. Overexpression of the cardiac $\mathrm{Na}^{+} / \mathrm{Ca}^{+}$exchanger increases susceptibility to ischemia/reperfusion injury in male, but not female, transgenic mice. Circ. Res. 83:1215-1223.

9. Weinberg, E.O., et al. 1999. Gender differences in molecular remodeling in pressure overload hypertrophy. J. Am. Coll. Cardiol. 34:264-273.

10. Kubota, T., et al. 1997. Dilated cardiomyopathy in transgenic mice with cardiac-specific overexpression of tumor necrosis factor-alpha. Circ. Res. 81:627-635.

11. Kubota, T., McTiernan, C.F., Frye, C.S., Demetris, A.J., and Feldman, A.M. 1997. Cardiac-specific overexpression of tumor necrosis factoralpha causes lethal myocarditis in transgenic mice. J. Card. Fail. 3:117-124.

12. Bryant, D., et al. 1998. Cardiac failure in transgenic mice with myocardial expression of tumor necrosis factor-alpha. Circulation. 97:1375-1381.

13. Franco, F., et al. 1999. Magnetic resonance imaging and invasive evaluation of development of heart failure in transgenic mice with myocardial expression of tumor necrosis factor-alpha. Circulation. 99:448-454

14. Kubota, T., et al. 1998. End-systolic pressure-dimension relationship of in situ mouse left ventricle. J. Mol. Cell. Cardiol. 30:357-363.

15. Kubota, T., et al. 2000. Soluble tumor necrosis factor receptor abrogates myocardial inflammation but not hypertrophy in cytokine-induced cardiomyopathy. Circulation. 101:2518-2525.

16. Preiss, J., et al. 1986. Quantitative measurement of sn-1,2-diacylglycerols present in platelets, hepatocytes, and ras- and sis-transformed normal rat kidney cells. J. Biol. Chem. 261:8597-8600.

17. Sugita, M., Willians, M., Dulaney, J.T., and Moser, H.W. 1975. Ceramidase and ceramide synthesis in human kidney and cerebellum. Description of a new alkaline ceramidase. Biochim. Biophys. Acta. 398:125-131.

18. Hess, H.H., and Derr, J.E. 1975. Assay of inorganic and organic phosphorus in the 0.1-5 nanomole range. Anal. Biochem. 63:607-613.

19. McTiernan, C.F., et al. 1997. Interleukin-1 beta inhibits phospholam- ban gene expression in cultured cardiomyocytes. Circ. Res. 81:493-503.

20. Thaik, C.M., Calderone, A., Takahashi, N., and Colucci, W.S. 1995. Interleukin-1 beta modulates the growth and phenotype of neonatal rat cardiac myocytes. J. Clin. Invest. 96:1093-1099.

21. Patten, M., Hartogensis, W.E., and Long, C.S. 1996. Interleukin-1beta is a negative transcriptional regulator of alpha1-adrenergic induced gene expression in cultured cardiac myocytes. J. Biol. Chem. 271:21134-21141.

22. Kolattukudy, P.E., et al. 1998. Myocarditis induced by targeted expression of the MCP-1 gene in murine cardiac muscle. Am. J. Pathol. 152:101-111.

23. Eghbali, M., Tomek, R., Sukhatme, V.P., Woods, C., and Bhambi, B. 1991. Differential effects of transforming growth factor-beta 1 and phorbol myristate acetate on cardiac fibroblasts. Regulation of fibrillar collagen mRNAs and expression of early transcription factors. Circ. Res. 69:483-490.

24. Brand, T., and Schneider, M.D. 1995. The TGF beta superfamily in myocardium: ligands, receptors, transduction, and function. J. Mol. Cell. Cardiol. 27:5-18.

25. Okumura, K., et al. 1998. Simultaneous quantitation of ceramides and 1,2-diacylglycerol in tissues by Iatroscan thin-layer chromatographyflame-ionization detection. Lipids. 33:529-532.

26. Li, Y.Y., Feng, Y.Q., Kadokami, T., McTiernan, C.F., and Feldman, A.M. 1999. Modulation of matrix metalloproteinase activities remodels myocardial extracellular matrix in TNF $\alpha$ transgenic mice. Circulation. 100:I752. (Abstr.)

27. Wiegmann, K., et al. 1992. Human 55-kDa receptor for tumor necrosis factor coupled to signal transduction cascades. J. Biol. Chem. 267:17997-18001.

28. Komi, J., and Lassila, O. 1998. Antioestrogens enhance tumour necrosis factor receptor 2 (TNF-R2) expression and TNF-R2-mediated proliferation in activated T cells. Scand. J. Immunol. 48:254-260.

29. Sunyer, T., Lewis, J., Collin-Osdoby, P., and Osdoby, P. 1999. Estrogen's bone-protective effects may involve differential IL-1 receptor regulation in human osteoclast-like cells. J. Clin. Invest. 103:1409-1418.

30. Roby, K.F., Laham, N., and Hunt, J.S. 1996. Cellular localization and steroid hormone regulation of mRNA encoding tumour necrosis factor receptor I in mouse uterus. J. Reprod. Fertil. 106:285-290. 